\title{
INFLUÊNCIA DA MINERALOGIA NO PROCESSO DE MOAGEM E QUALIDADE DA PELOTA COM USO DE DATA ANALYTICS*
}

Catia Casagrande ${ }^{1}$

Bernardo Libardi'

Rossano Pilon ${ }^{3}$

\section{Resumo}

O processo de pelotização surgiu na década de 40. Até hoje, as qualidades químicas e físicas são utilizadas como controle e monitoramento do processo. Entretanto, a característica mineralógica e microestrutural do minério de ferro mostra grande importância para ajustes no processo, a fim de melhoria da qualidade do produto e redução de custo. As usinas de pelotização em Tubarão recebem minérios de várias minas do Quadrilátero Ferrífero, dificultando a caracterização de todas as misturas que entram nas mesmas. Com o intuito de obter maior eficiência nos dados de características do minério de ferro e processo, o uso de um software de estatística pode auxiliar no entendimento do comportamento do minério de ferro nas operações unitárias. Neste estudo, o objetivo foi relacionar à qualidade física do produto de moagem e pelota queimada por meio dos modelos preditivos, onde foi utilizado os tipos de minas, mineralogia e microestrutura como preditores. Com a análise realizada pela simulação e o conhecimento prático do processo foi possível correlacionar o efeito da mineralogia na qualidade física do produto do processo de moagem e qualidade física da pelota.

Palavras-chave: Minério de ferro; Pelotização; Mineralogia; Data Analytics.

\section{INFLUENCE OF THE MINERALOGY IN GRIDING PROCESS AND PELLET QUALITY USING DATA ANALYTICS}

\section{Abstract}

The pelletizing process appeared in the 40's. To now, the chemical and physical qualities are used as process control and monitoring. However, the mineralogical characteristics of iron ore show great importance for process adjustments in order to improve product quality and reduce costs. The pelletizing plants in Tubarão receive iron ores from several mines from Iron Quadrangle, making it difficult to characterize all the mixtures that enter them. In order to obtain greater efficiency in the iron ore characteristics and process data, the use of statistical software can help in understanding the behavior of iron ore in unit operations. In this study, the objective was to relate to the physical quality of the grinding product and pellet induration through the predictive models, where the types of mines and mineralogy were used as predictors. With the analysis carried out by the simulation and the practical knowledge of the process it was possible to correlate the effect of the mineralogy on the products physical quality of the grinding process and the pellet physical quality.

Keywords: Iron ore; Pelletizing; Mineralogical; Data Analytics.

1 Engenheira Geóloga, Mestre, Engenheira Sênior, Diretoria de Pelotização, Vale S.A., Vitória, ES, Brasil.

2 Engenheiro Químico, Diretoria de Pelotização, Vale S.A., Vitória, ES, Brasil.

3 Engenheiro Metalúrgico, Engenheiro Pleno, Diretoria de Pelotização, Vale S.A., Vitória, ES, Brasil. 


\section{INTRODUÇÃO}

O processo de aglomeração de finos de minério de ferro é conhecido desde a década de 40 , nomeado de pelotização. Atualmente a Vale opera nove usinas de pelotização, a maioria localizada no Brasil, sendo uma em Omã. A produção de pelotas em 2016 foi de $46.7 \mathrm{Mta}$.

A matéria prima principal para produção de pelota é o minério de ferro, com $97 \%$ na composição total, posteriormente o aglomerante, fundente e combustível sólido. 0 controle das características de minério de ferro é realizado pelas análises química e física em toda a cadeia, desde a mina até a pelotização. Entretanto, vários estudos mostram a influência da mineralogia no processo mineral [1] e processo metalúrgico [2-11], evidenciando a necessidade desta análise tecnológica.

Apesar do grande número de trabalhos científicos relacionados aos estudos geometalúrgicos, a presença de variáveis mineralógicas e metalúrgicas em modelos de recursos de minério de ferro não é muito comum. Nesta linha técnica, a Vale busca a cada dia, o entendimento do efeito da mineralogia nos processos de ferrosos em toda a cadeia produtiva.

Minérios de ferro oriundos de diversas minas com diferentes gêneses são enviados para a pelotização em Tubarão, Vitória, a fim de aglomerar e enriquecer o produto final. A previsibilidade mineralógica e a influência dos minerais nas operações unitárias da pelotização são de suma importância para a otimização do processo, melhoria da qualidade do produto e redução dos custos operacionais.

As principais operações unitárias na pelotização são: moagem, filtragem, pelotamento e queima. $O$ processo de moagem recebe os minérios de ferro processados pelas usinas de concentração de tratamento de minério de ferro, localizadas no Quadrilátero Ferrífero. As qualidades físicas do produto deste processo, tais como, superfície específica e granulometria, são pré-estabelecidas para continuação das etapas seguintes. Além disso, o custo energético nesta etapa é considerado um dos mais altos em todo o processo de pelotização [2,5,9].

A qualidade física da pelota queimada (resistência à compressão) é usada como especificação do produto e pelo controle interno do processo, pois caso necessário, os ajustes operacionais são realizados para adequação. Por isso, conhecer os parâmetros operacionais que influenciam nestas informações é fundamental para melhoria no desempenho do processo e tomadas de decisões da operação.

Os avanços na área de tecnologia da informação deram oportunidades para aplicar os conceitos de mineração e análise avançada de dados no desenvolvimento de modelos preditivos para os processos das plantas industriais [12-14]. Com o intuito de obter maior eficiência na avaliação dos dados de características mineralógica do minério de ferro e processo, foi utilizado o software estatístico da IBM, SPSS Modeler para a construção de modelos.

Entre as diversas técnicas empregadas na análise de dados, a clusterização e a modelagem por redes neurais artificiais são consideradas muito eficientes. A clusterização é uma técnica que, por meio de ferramentas estatísticas, busca 
agrupar uma série de dados de acordo com as características comuns entre as variáveis. Enquanto que as redes neurais artificiais são modelos desenvolvidos a partir do entendimento do funcionamento do cérebro humano. $O$ algoritmo simula a transmissão de sinais através de neurônios, os quais atribuem pesos para as variáveis de entrada, e determinam um valor de saída esperado para a variável alvo.

O objetivo principal deste trabalho é avaliar as variáveis do processo, tipos de minas e características mineralógica / microestrutural do minério de ferro em relação à qualidade física do produto de moagem (ganho de granulometria e superfície específica) e pelota queimada (resistência à compressão) por meio de redes neurais artificiais.

\section{MATERIAIS E MÉTODOS}

Os minérios de ferro das cinco minas que abastecem as usinas de pelotização foram submetidos às análises de mineralogia e de microestrutura em microscópio ótico. A maioria das análises foram realizadas no Centro de Pesquisas Tecnológicas (CPT) em Mariana e Centro de Tecnologia de Ferrosos (CTF) no Miguelão, ambos em Minas Gerais. A metodologia adotada para esta técnica foi de contagem de partículas de minerais.

As análises de superfície específica (SE) foram realizadas no equipamento Blaine Star e granulometria, passante em 0.045mm, no Alpine. Ambas as técnicas foram feitas para pilhas que alimenta a moagem e pós- moagem. A análise de resistência à compressão da pelota queimada foi realizada no equipamento Lloyd instruments modelo LF plus.

A planilha de previsibilidade de mineralogia e microestrutura foi elaborada pela média ponderada das participações dos minérios de ferro e os resultados de análise de microscopia ótica de cada tipo de minério. Os minerais analisados foram hematitas (granular, tabular, lobular, martita e microcristalina), goethita e magnetita. Também foram avaliados os minerais compactos (granular, tabular) e minerais porosos (lobular, martita, microcristalina e goethita).

O levantamento dos dados de previsibilidade de mineralogia e parâmetros operacionais de moagem e queima foram realizados no período de 01/01/16 a 19/02/17, sendo coletados médias diárias nos sistemas automatizados da Vale.

A tabela 1 mostra os dados operacionais de uma usina de pelotização e as respectivas siglas.

Tabela 1. Abreviações dos parâmetros das usinas

\begin{tabular}{lc}
\hline Siglas & Descrição \\
\hline MIN_ & Mina \\
\hline SE_MA & Superfície específica minério alimentado \\
\hline Gran_MA & Granulometria $(\%<0.045 \mathrm{~mm})$ minério alimentado \\
\hline Pot_Moa & Potência de moagem \\
\hline Prod_Moa & Produção de moagem \\
\hline Pres_HC & Pressão do hidrociclone \\
\hline Gran_PP & Granulometria $(\%<0.045 \mathrm{~mm})$ minério prensado \\
\hline Temp_Req_Direta & Temperatura ar de recuperação \\
\hline
\end{tabular}




\begin{tabular}{lc}
\hline Ind_Gran_PQ & Índice granulométrico \\
\hline Temp_CV & Temperatura caixas de vento \\
\hline Ret_Pel & Retorno do Pelotamento \\
\hline SE_PP & Superfície específica minério prensado \\
\hline $\mathrm{MgO}$ PR & Quantidade de MgO na polpa prensada \\
\hline $\mathrm{H}_{2} \mathrm{O}$ PR & Umidade da polpa retida \\
\hline Num_Quedas & Número de quedas da pelota verde \\
\hline$-5 \_P Q$ & Fração de finos de pelota queimada \\
\hline
\end{tabular}

Alguns filtros foram usados para eliminação dos outliers, tais como, produção de moagem, produção de pelota queimada, pilhas atípicas de recebimento e funcionamento de equipamentos.

Os dados foram processados pelo software SPSS Modeler da IBM, onde a clusterização foi feita com algoritmos K-means e Two-step. Foi feito modelagem por redes neurais com algoritmo de MPL (Multiple Layer Perceptron) e o treinamento das redes foi realizado de forma padrão ou com o aprimoramento de estabilidade (bagging) e de exatidão (boosting) [12,14].

\section{RESULTADOS E DISCUSSÃO}

\subsection{Moagem}

A Figura 1 apresenta o gráfico dos tamanhos dos clusters, de acordo a quantidade de pilhas, e a tabela com participações das minas em cada cluster.

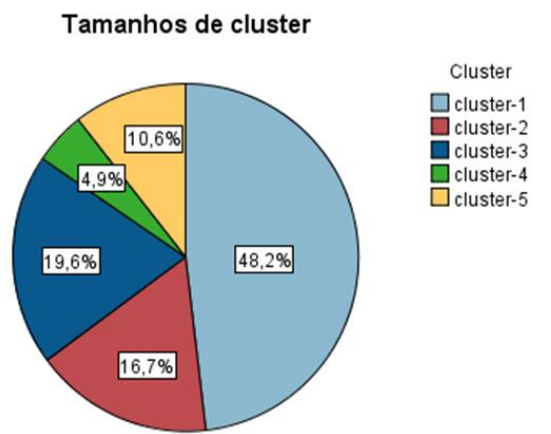

\begin{tabular}{lccccc}
\hline Campo & Cluster 1 & Cluster 2 & Cluster 3 & Cluster 4 & Cluster 5 \\
\hline MIN_A & $48.4 \%$ & $45.4 \%$ & $39.3 \%$ & $37.0 \%$ & $33.6 \%$ \\
\hline MIN_B & $49.8 \%$ & $46.6 \%$ & $50.2 \%$ & $49.9 \%$ & $60.0 \%$ \\
\hline MIN_C & $0.0 \%$ & $0.0 \%$ & $6.2 \%$ & $0.0 \%$ & $0.0 \%$ \\
\hline MIN_D & $0.0 \%$ & $0.0 \%$ & $0.0 \%$ & $7.5 \%$ & $0.0 \%$ \\
\hline MIN_E & $1.3 \%$ & $7.4 \%$ & $3.6 \%$ & $4.5 \%$ & $6.10 \%$ \\
\hline
\end{tabular}

Figura 1. Gráfico de tamanho dos clusters e participação das minas por clusters.

O cluster 1 é o maior em relação aos demais, enquanto que o cluster 4 apresenta a menor quantidade de eventos. O cluster de menor tamanho foi gerado devido a presença exclusiva do minério da mina $D$. Mesmo fato observado no cluster 3 , com a presença do minério da mina $\mathrm{C}$.

A Figura 2 apresenta as informações dos clusters de mineralogia /microestrutura. 
Tamanhos de cluster

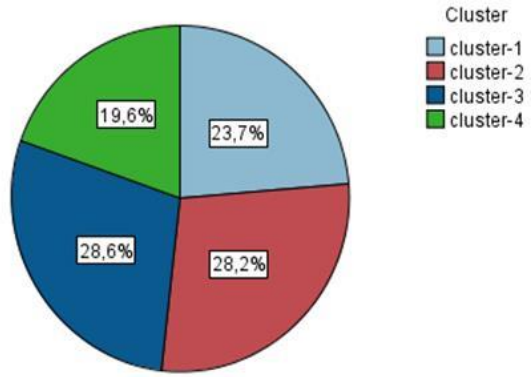

\begin{tabular}{ccccc}
\hline Campo & Cluster 1 & Cluster 2 & Cluster 3 & Cluster 4 \\
\hline Hematita Tabular & $47.4 \%$ & $36.5 \%$ & $37.3 \%$ & $35.1 \%$ \\
\hline Hematita granular & $43.7 \%$ & $54.7 \%$ & $49.9 \%$ & $50.7 \%$ \\
\hline Hematita lobular & $1.3 \%$ & $0.9 \%$ & $1.1 \%$ & $3.8 \%$ \\
\hline Goethita & $3.0 \%$ & $3.0 \%$ & $4.4 \%$ & $4.0 \%$ \\
\hline Martita & $1.6 \%$ & $1.8 \%$ & $3.6 \%$ & $2.0 \%$ \\
\hline Magnetita & $0.7 \%$ & $0.8 \%$ & $1.4 \%$ & $1.0 \%$ \\
\hline Microcristalina & $0.0 \%$ & $0.0 \%$ & $0.0 \%$ & $0.9 \%$ \\
\hline
\end{tabular}

Figura 2. Cluster de tamanho de clusters e participação de mineralogia/microestrutura por clusters.

Nota-se que todos os clusters apresentam maior participação de hematita tabular e granular, em relação aos outros minerais de ferro. A presença de microcristalina no minério de ferro foi responsável pela diferenciação do cluster 4.

Em relação as microestruturas, o software apresentou 4 clusters com tamanhos similares. O cluster 1 apresentou maior presença de minerais porosos (11.3\%), enquanto o cluster 3 maior participação de hematitas compactas (92.1\%).

A Figura 3 mostra a importância dos preditores, entre eles os tipos de minas, no modelo de delta de granulometria da moagem (diferença entre a polpa pós moagem e minério alimentado).

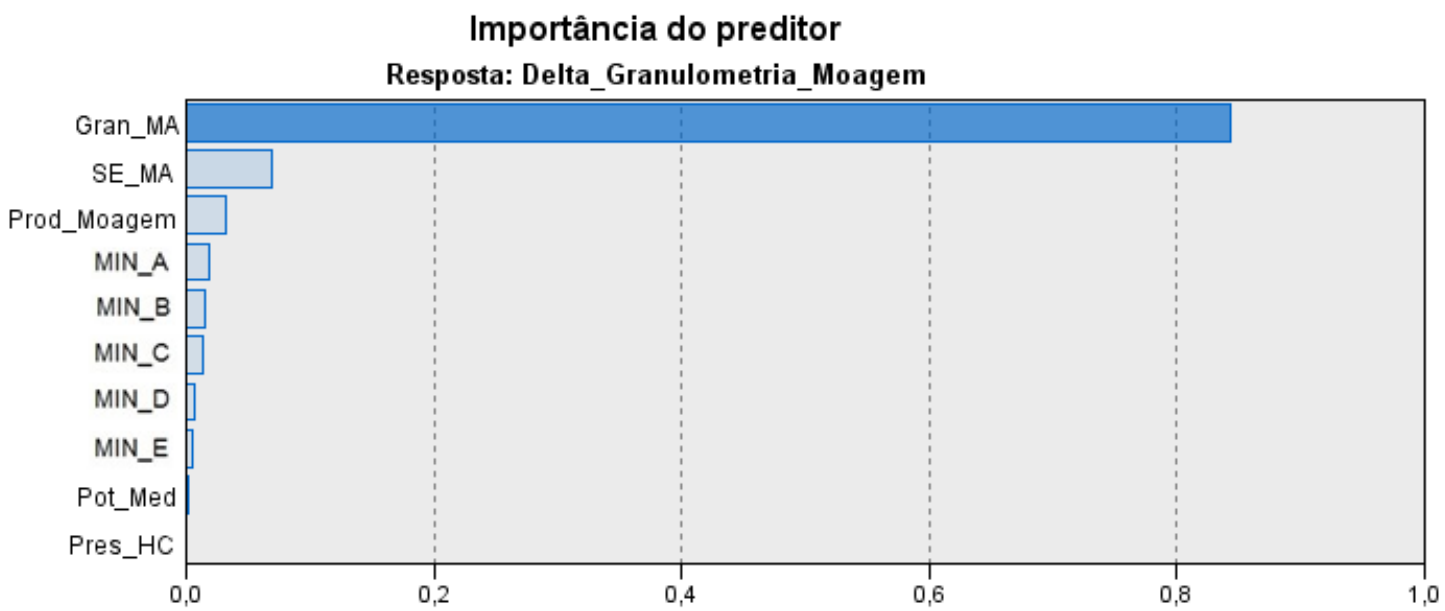

Figura 3. Importância dos preditores na resposta de delta de granulometria na moagem.

$\mathrm{Na}$ análise realizada pelo software, a granulometria do minério alimentado é o fator mais importante para o ganho de granulometria, seguido pela superfície específica inicial e produção de moagem. Os tipos de minas e potência da moagem mostraram pequena importância para o delta da granulometria.

Os modelos construídos com a mineralogia e microestrutura do minério em relação à importância dos preditores apresentaram resultados semelhantes aos resultados da Figura 3. 
A Figura 4 mostra a correlação da variável resposta com os preditores, entre eles, os tipos de minas.

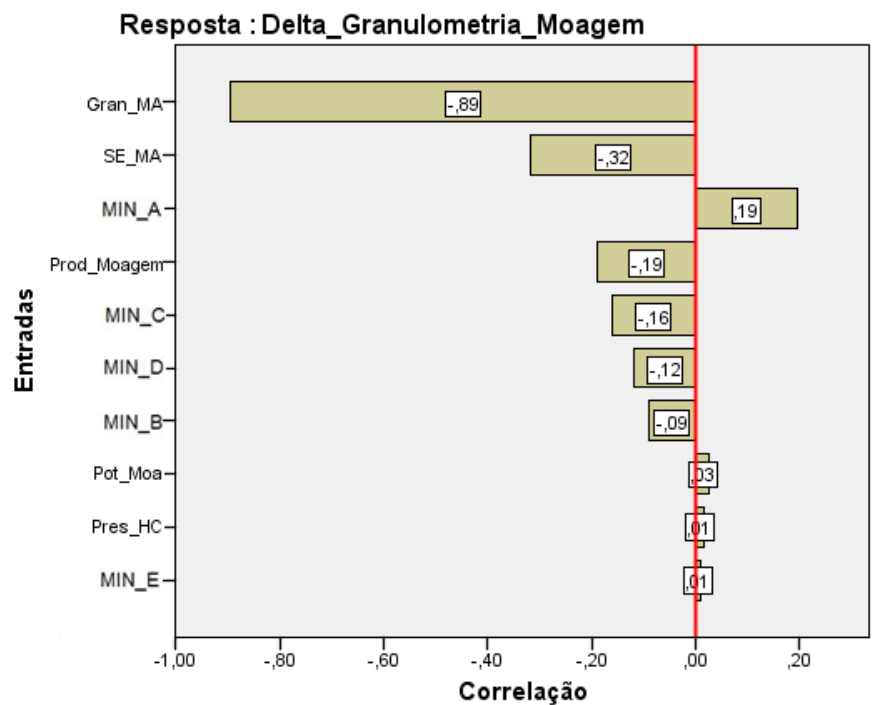

Figura 4. Correlação entre o delta de granulometria na moagem e preditores.

A Figura 4 apresenta que a maioria dos preditores mostra correlações negativas, por exemplo, quanto maior a granulometria de entrada da mistura, menor será o delta na moagem. Este mesmo efeito pode ser observado para superfície específica de alimentação e produção da moagem. Os minérios de ferro das minas A influencia positivamente no ganho de moagem, enquanto dos demais apresentam correlações negativas.

A Tabela 2 mostra a importância dos preditores, com a inclusão dos tipos de minas, mineralogia e microestrutura, nos modelos de delta de superfície específica da moagem.

Tabela 2. Importância dos preditores no ganho de superfície específica da moagem.

\begin{tabular}{|c|c|c|c|c|c|}
\hline \multicolumn{2}{|c|}{ Delta SE x Mineralogia } & \multicolumn{2}{|c|}{ Delta SE x Mina } & \multicolumn{2}{|c|}{ Delta SE x Microestrutura } \\
\hline Preditor & Importância & Preditor & Importância & Preditor & Importância \\
\hline Se_MA_Med & 0,19 & MIN_B & 0,25 & Hematita compacta & 0,35 \\
\hline Hematita tabular & 0,14 & MIN_A & 0,18 & Minerais porosos & 0,3 \\
\hline Hematita lobular & 0,14 & Se_MA_Med & 0,14 & Se_MA_Med & 0,2 \\
\hline Hematita microcirstalina & 0,1 & Pot_Med_Moa & 0,12 & Gran_MA_Med & 0,07 \\
\hline Martita & 0,09 & Pres_HC_Med & 0,07 & Pot_Moa & 0,05 \\
\hline Goethita & 0,08 & Prod_Moagem & 0,06 & Prod_Moagem & 0,02 \\
\hline Pot_Med_Moa & 0,07 & MINE & 0,05 & Pres_HC & 0,01 \\
\hline Pres_HC_Med & 0,06 & Gran_MA_Med & 0,05 & & \\
\hline Prod_Moagem & 0,06 & MIN_D & 0,04 & & \\
\hline Gran_MA_Med & 0,04 & MIN_C & 0,04 & & \\
\hline Hematita granular & 0,03 & & & & \\
\hline
\end{tabular}

Os modelos de delta de superfície especifica mostraram maior dependência das variáveis mineralógicas quando comparados com os modelos de granulometria. 
A Figura 5 mostra a correlação do delta de superfície específica com os preditores, entre eles, os tipos de minas.

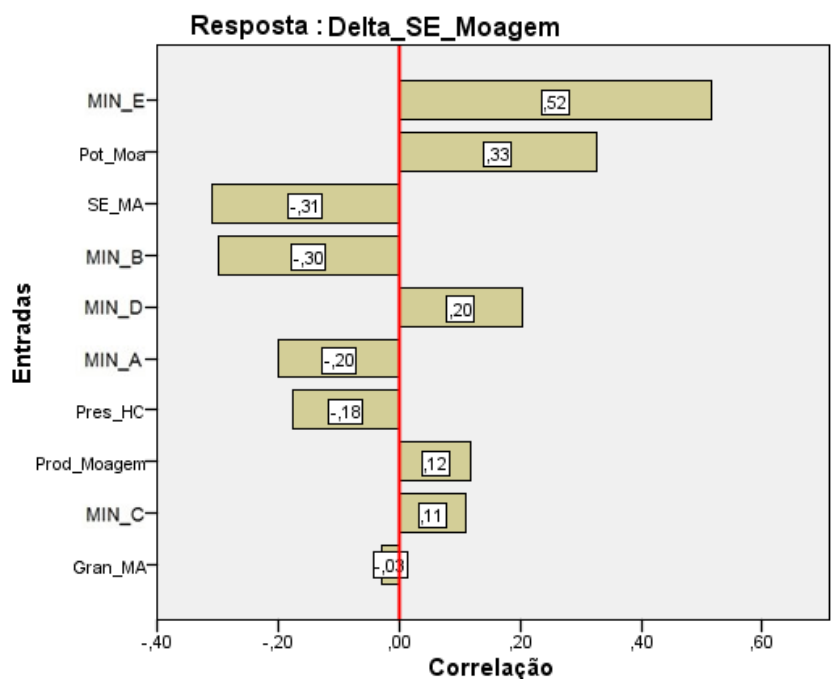

Figura 5. Correlação do delta de SE da moagem com as minas entre os preditores.

Os minérios de ferro das minas $E, D$ e $C$ mostraram que quanto maior a participação deles, maior o delta de SE. Este efeito também ocorre em relação à potência do moinho e produção de moagem. Enquanto que o aumento da granulometria e SE iniciais reduzem o resultado de delta de SE.

A correlação do delta de SE com os preditores, incluindo as mineralogias/microestrutura, pode ser observada no gráfico da Figura 6.

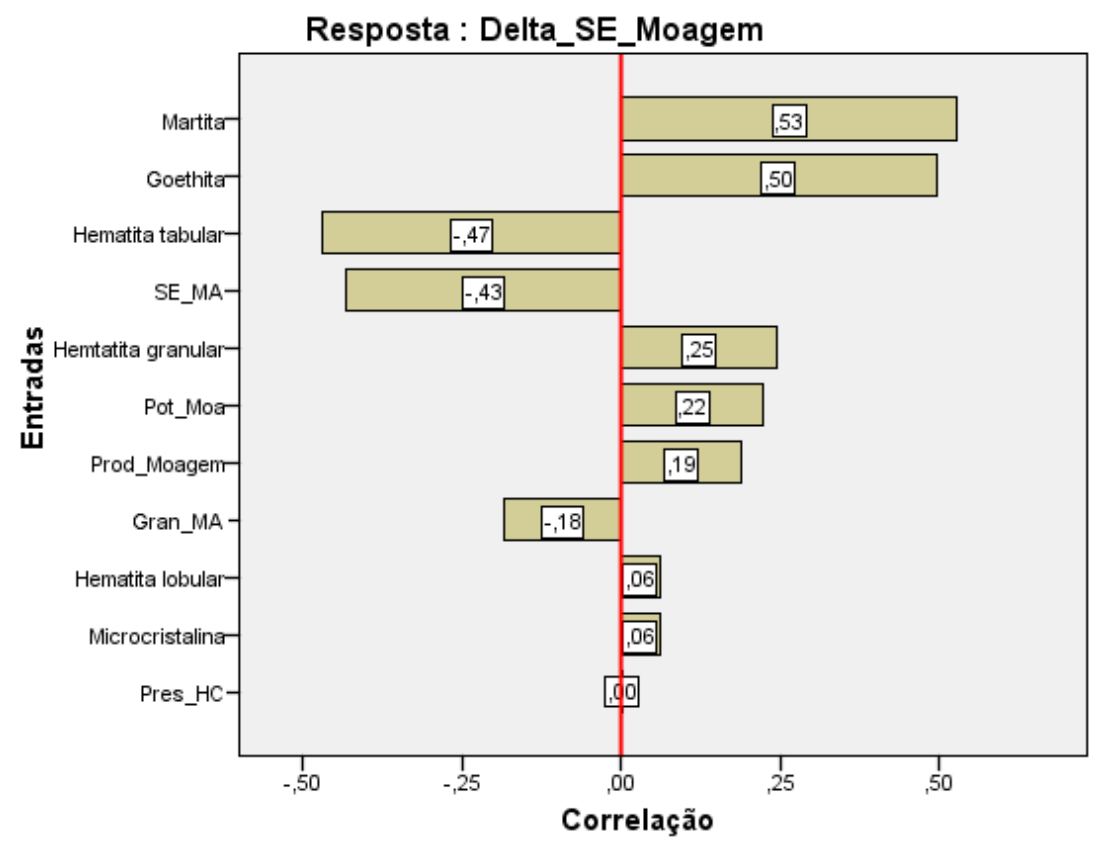

Figura 6. Correlação do delta de SE da moagem com as mineralogias entre os preditores.

Os minerais goethita e martita apresentam correlações diretas em relação ao delta de superfície específica, enquanto que a hematita tabular mostra o inverso. A hematita granular deveria ter o mesmo comportamento da hematita tabular, pois 
ambos são minerais compactos, porém apresentou o oposto. Aprofundando neste efeito, foi visto que a microestrutura da hematita granular era policristalina, beneficiando a cominuição da partícula. A superfície específica e granulometria iniciais mostraram o mesmo comportamento que o modelo de granulometria, impactando negativamente no ganho de qualidade física.

Ao analisar o efeito da mineralogia no processo de moagem, foi visto que na prática a análise mais representativa e factível seria a relação entre minerais porosos e hematita compacta. Avaliando estes dois preditores em relação ao ganho de SE, notou-se que eles mostram importância muito maior do que os parâmetros operacionais de moagem (produção e potência) como também granulometria e superfície específica iniciais. A Figura 7 mostra a correlação entre estes preditores e a variável resposta.

É fato que os minerais porosos ajudam no aumento de produção, e pela análise foi visto que quanto maior a participação de minerais porosos, potência e produção, maior o ganho de superfície específica. Enquanto que as hematitas compactas, superfície específica e granulometria de alimentação têm efeito contrário.

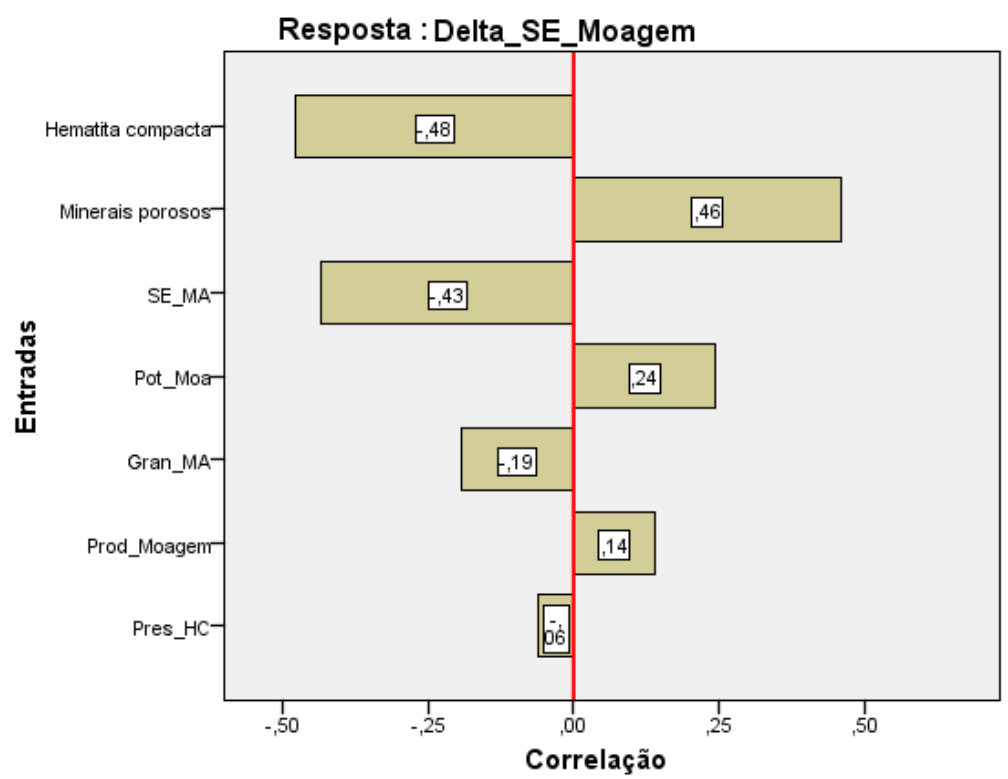

Figura 7. Correlação do delta de SE da moagem com a microestrutura entre os preditores.

A figura 8 mostra a importância dos preditores no modelo de delta de superfície específica utilizando os clusters de microestrutura como preditores 


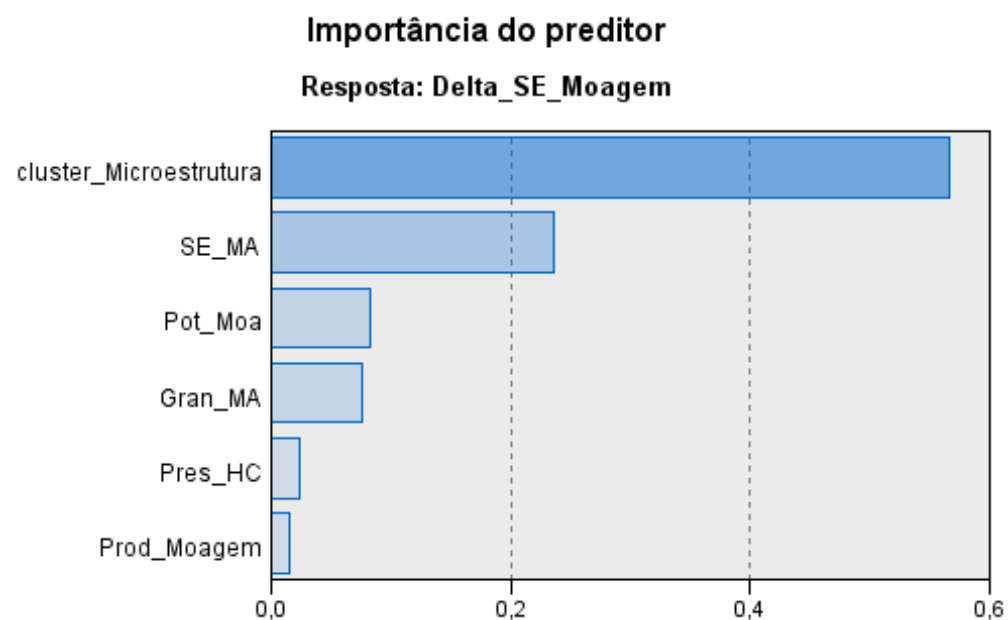

Figura 8. Importância dos preditores na resposta de delta de granulometria na moagem.

A variável de maior importância para o modelo de delta de SE é o cluster de microestrutura, seguido pela superfície específica do minério alimentado. Essa informação aponta para uma grande dependência do processo de moagem com o mistura de minérios. A figura 9 apresenta as curvas de delta de SE para cada um dos cenários de microestrutura com a variação da potência da moagem em diferentes níveis de produção.
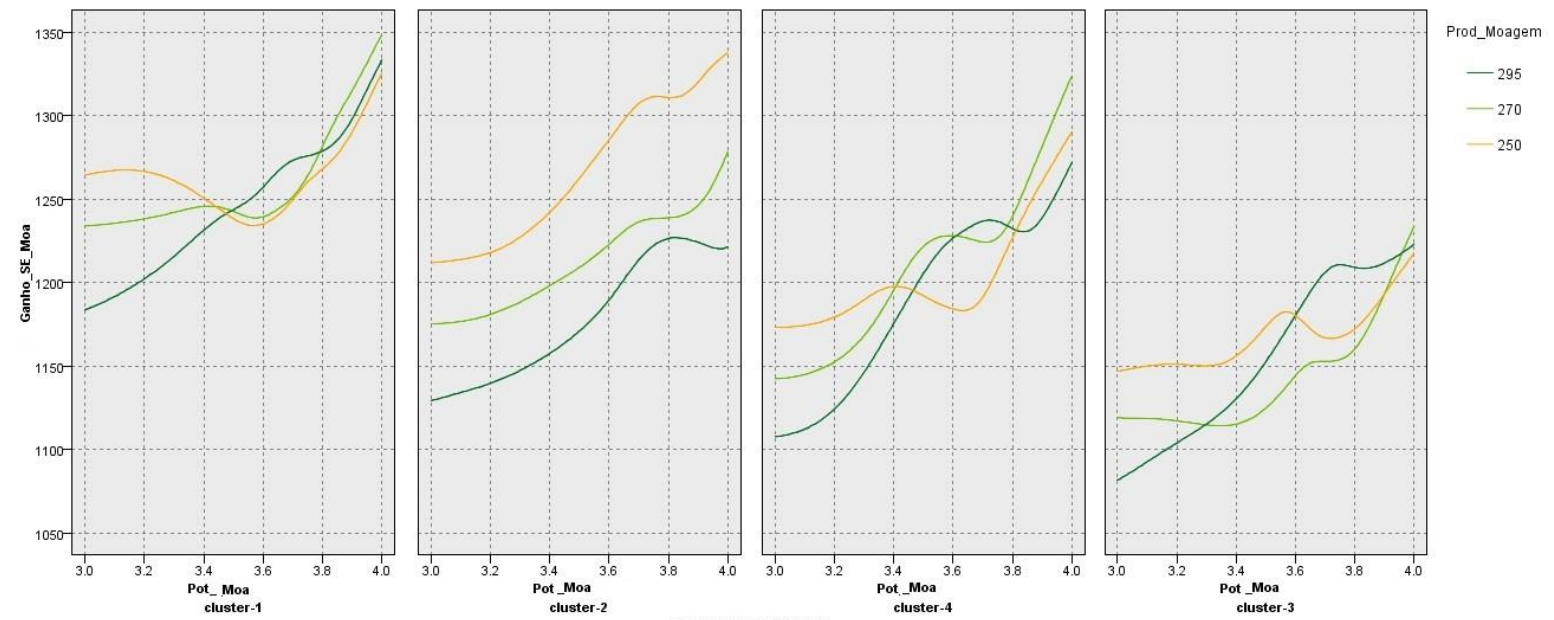

Figura 9. Ganho de superfície específica na moagem com a variação da potência da moagem para os clusters de microestrutura.

As curvas apresentam um decréscimo da eficiência da moagem à medida que são utilizados misturas de minérios com maiores frações de minerais compactos. Para todos os cenários o aumento da potência leva a um maior delta de superfície, sendo que as curvas apresentam comportamentos diferentes para mudanças de taxas de alimentação.

\subsection{Queima}

Além das variáveis ligadas ao processo de moagem, foi possível avaliar o efeito da mineralogia na qualidade física. A Figura 10 apresenta a importância dos preditores no modelo de resistência à compressão da pelota queimada. 


\section{Importância do preditor}

Resposta: MCOMP US 5

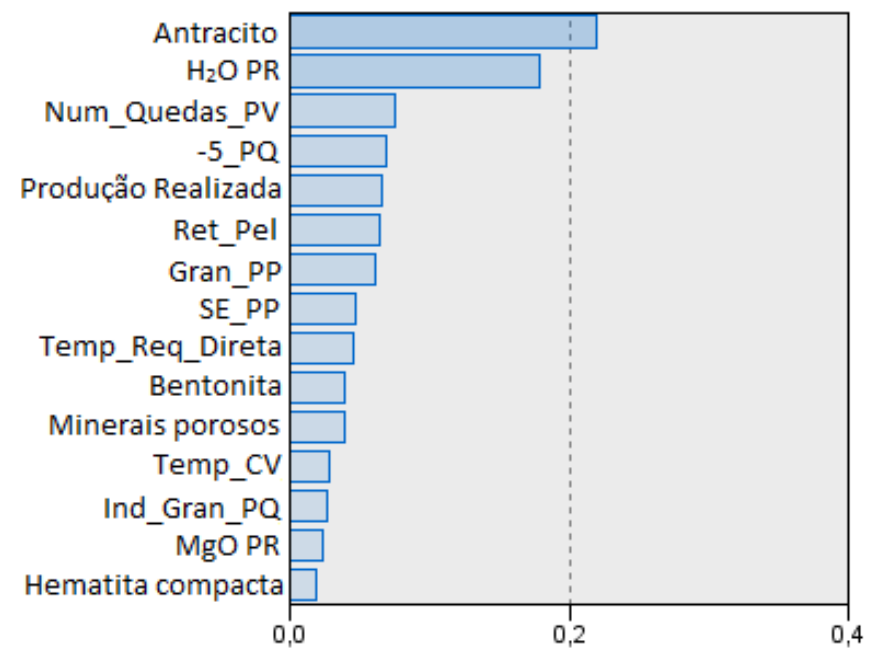

Figura 10. Importância dos preditores na resposta de resistência a compressão da pelota.

Os principais preditores no modelo da resistência à compressão são ligados às variáveis operacionais.

Entre as variáveis mais importantes para a construção do modelo, o antracito, fração de finos menores que $5 \mathrm{~mm}$ e produção de pelotas queimadas são diretamente relacionadas ou impactadas pela operação do forno. Enquanto as variáveis umidade da polpa retida, número de quedas da pelota verde e retorno do pelotamento estão ligadas a operações unitárias anteriores ao forno. As variáveis mineralógicas se mostraram pouco relevantes para a construção do modelo.

O efeito de cada uma das variáveis na resposta do modelo de resistência à compressão pode ser observado na Figura 11.

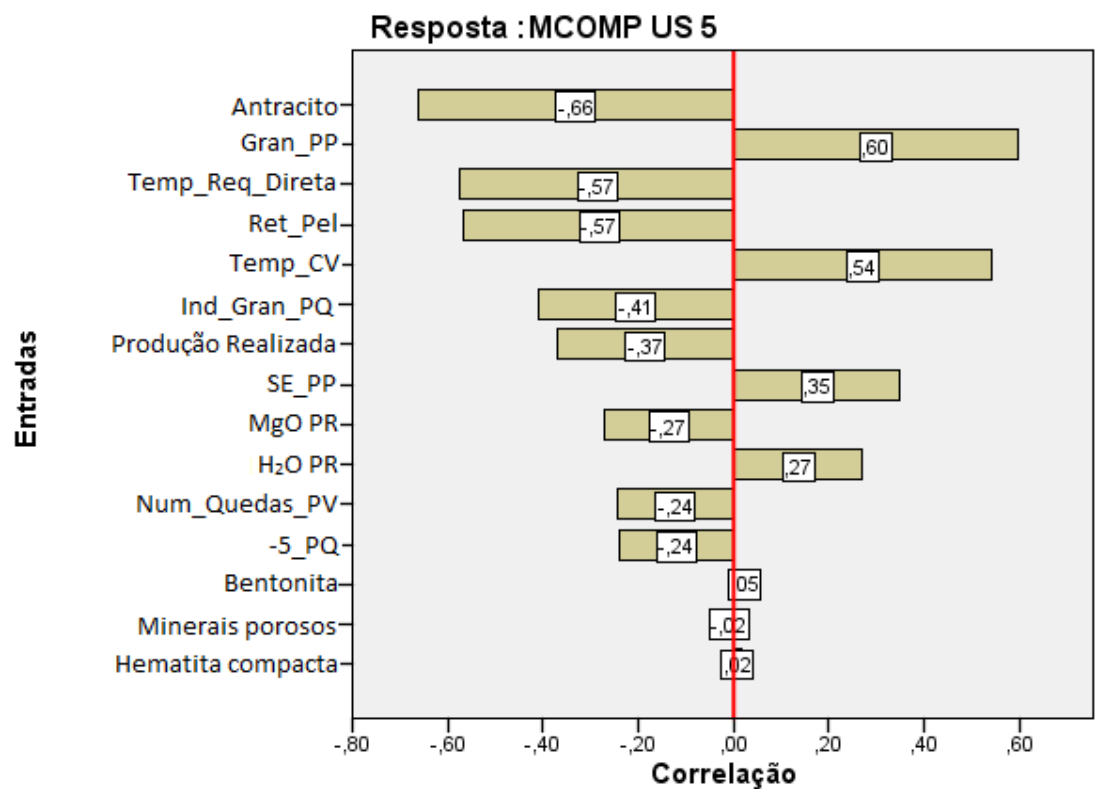

Figura 11. Correlação da resistência à compressão da pelota com os preditores. 
A dosagem de antracito praticada na planta visa reduzir o consumo dos demais combustíveis queimados no forno, os níveis adotados atualmente impactam negativamente na resistência à compressão quando da sua elevação.

A elevação da temperatura do gás recuperado está relacionada com o calor recuperado do leito de pelotas no resfriamento e é função da dosagem de antracito também.

O retorno do pelotamento, índice granulométrico, e número de quedas impactam negativamente a compressão quando da sua elevação. Uma piora das condições do pelotamento, maior deformidade da distribuição granulométrica e maior plasticidade da pelota tendem a queimar de forma menos uniforme no leito, o que reduz a média de resistência à compressão da pelota.

A granulometria e superfície da polpa que alimenta o pelotamento são diretamente proporcionais à resistência à compressão [15]. A temperatura das caixas de vento da região de queima é diretamente proporcional à elevação da resistência à compressão. Isto pode significar uma transferência de calor mais intensa para o leito.

\section{CONCLUSÃO}

Os preditores de mineralogia e microestrutura não mostraram importância para o ganho granulometria na moagem. Foi possível ver correlação entre o ganho de granulometria com os preditores de parâmetros de moagem e tipos de minas.

Os preditores de mineralogia, microestrutura e tipos de minas mostraram importância para o ganho de superfície específica na moagem.

O modelo de ganho de superfície específica na moagem com preditores de parâmetros de moagem e microestrutura foi considerado o melhor.

O modelo de resistência à compressão mostra a forte influência da dosagem de antracito e produção o que condiz com a teoria [4]. As demais variáveis como retorno do pelotamento, índice granulométrico e granulometria PP também se mostram importantes, pois influenciam a qualidade da pelota crua que é enfornada.

\section{Agradecimentos}

Ao colega Ricardo Olympio pelas contribuições técnicas.

\section{REFERÊNCIAS}

1 Santos L e Brandão P. "Variações Mineralógicas, Morfológicas e Microestruturais em minérios de ferro do Quadrilátero Ferrífero, MG e suas Implicações no Processo Mineral." In 4th Simpósio Brasileiro de Minério de Ferro. 2003; pp. 674-683.

2 Ribeiro, M. R. Investigação das Características dos Minérios de Ferro da CVRD que Influenciam seu Comportamento na Moagem. Escola de Engenharia, Universidade Federal de Ouro Preto. Dissertação de Mestrado. 2004; 70p. 
Mourão J.M. \& Stegmiller L. Influência da estrutura dos minérios de ferro na sua moabilidade. In: encontro nacional de tratamento de minérios e hidrometalurgia. Anais, 1990, 1: 228-243.

4 Meyer, M. M. Processo de Moagem e Formação de Fases em Pelotas de Minério de Ferro. Departamento de Geologia, Universidade Federal de Ouro Preto, Ouro Preto, Dissertação de Mestrado. 2016; 86p.

5 Graça L. M., Lagoeiro L. E., Lima R. M. F., Barbosa P. F., Meyer Machado M. Effect of the morphological types in grinding of iron-ore products. Mineral Processing and Extractive Metallurgy Review: An International Journal. 2015, 36:5, 324-331.

6 Graça L.M.; Alvarenga, T.F. Mineralogia de alimentação das Usinas Vale I e II. In: Seminário Brasileiro de Minério de Ferro. Anais. 2009, 2:350-356.

7 Graça L. M. Implicações das propriedades mineralógicas e microestruturais do minério de ferro no processo industrial de pelotização. Departamento de Geologia, Universidade Federal de Ouro Preto. Tese de Doutorado. 2015, 204p.

8 Chagas T. T. R. A moabilidade na moagem secundária de pellet feeds de minérios de ferro em função da mineralogia, química e microestrutura. Ouro Preto, Rede Temática em Engenharia de Materiais, Escola de Minas, Universidade Federal de Ouro Preto, Dissertação de Mestrado. 2008, 74p.

9 Casagrande, C.; Vieira, M. B.; Guachalla, S.; Mafra, W. Influência da Mineralogia no Processo de Pelotização de Minérios de Ferro. 43ํㅗㄴ Seminário de Redução de Minério de Ferro e Matérias-Primas, Belo Horizonte, 2013.

10 Sá $\mathrm{K}$ et al. Efeito da Composição Mineralógica na Resistência à Compressão de Pelotas de Minério de Ferro. Tecnologia em Metalurgia e Materiais, São Paulo. 2004; v. 1, n.2, pp 18-22.

11 Graça L et al. "Effect of the Morphological Types in Grinding of Iron-Ore Products." Minerals Processing and Extractive Mettalurgy Review: An International Journal. 2015; 36:5, 324-331.

12 McCormick, K et al. IBM SPSS Modeler Cookbook; ed: Packt Publishing. 2013; 353p.

13 Hastie, $\mathrm{T}$ et al. The Elements of Statistical Learning: Data Mining, Inference, and Prediction. $2^{\circ}$ edition: Springer. 2008; 727p.

14 Wendler T.; Gröttrup S. Data Mining with SPSS Modeler: Theory, Exercises and Solutions. Springer. 2016. 1059p. 